ESAIM: M2AN 47 (2013) 149-167

DOI: $10.1051 / \mathrm{m} 2 \mathrm{an} / 2012026$
ESAIM: Mathematical Modelling and Numerical Analysis

www.esaim-m2an.org

\title{
A GENERAL SEMILOCAL CONVERGENCE RESULT FOR NEWTON'S METHOD UNDER CENTERED CONDITIONS FOR THE SECOND DERIVATIVE
}

\author{
José Antonio Ezquerroํㅜ, Daniel González ${ }^{1}$ and Miguel Ángel Hernández ${ }^{1}$
}

\begin{abstract}
From Kantorovich's theory we present a semilocal convergence result for Newton's method which is based mainly on a modification of the condition required to the second derivative of the operator involved. In particular, instead of requiring that the second derivative is bounded, we demand that it is centered. As a consequence, we obtain a modification of the starting points for Newton's method. We illustrate this study with applications to nonlinear integral equations of mixed Hammerstein type.
\end{abstract}

Mathematics Subject Classification. 45G10, 47H99, 65J15.

Received November 3, 2011. Revised March 26, 2012.

Published online July 31, 2012.

\section{INTRODUCTION}

As is well-known, many scientific and engineering problems can be brought in the form of a nonlinear equation. Although some equations can be solved analytically, we usually look for numerical approximations of the solutions, since finding exact solutions is usually difficult. To approximate a solution of a nonlinear equation we normally use iterative methods and Newton's method is one of the most used because of its simplicity, easy implementation and efficiency.

To give sufficient generality to the problem of approximating a solution of a nonlinear equation by Newton's method, we consider equations of the form $F(x)=0$, where $F$ is a nonlinear operator, $F: \Omega \subseteq X \rightarrow Y$, defined on a non-empty open convex domain $\Omega$ of a Banach space $X$ with values in a Banach space $Y$, which is usually known as the Newton-Kantorovich method and whose algorithm is

$$
\left\{\begin{array}{l}
x_{0} \text { given in } \Omega, \\
x_{n}=x_{n-1}-\left[F^{\prime}\left(x_{n-1}\right)\right]^{-1} F\left(x_{n-1}\right), \quad n \in \mathbb{N} .
\end{array}\right.
$$

The first semilocal convergence result for Newton's method in Banach spaces is due to L.V. Kantorovich, which is usually known as the Newton-Kantorovich theorem and is proved under the following conditions for

\footnotetext{
Keywords and phrases. Newton's method, the Newton-Kantorovich theorem, semilocal convergence, majorizing sequence, a priori error estimates, Hammerstein's integral equation.

1 University of La Rioja, Department of Mathematics and Computation, C/ Luis de Ulloa s/n, 26004 Logroño, Spain.

<jezquer><daniel.gonzalez><mahernan>@unirioja.es
} 
the operator $F$ and the starting point $x_{0}$ :

(i) there exist $\Gamma_{0}=\left[F^{\prime}\left(x_{0}\right)\right]^{-1} \in \mathcal{L}(Y, X)$, for some $x_{0} \in \Omega$, with $\left\|\Gamma_{0}\right\| \leq \beta$ and $\left\|\Gamma_{0} F\left(x_{0}\right)\right\| \leq \eta$, where $\mathcal{L}(Y, X)$ is the set of bounded linear operators from $Y$ to $X$;

(ii) $\left\|F^{\prime \prime}(x)\right\| \leq k$ for $x \in \Omega$;

(iii) $k \beta \eta \leq \frac{1}{2}$.

Since then many papers have appeared that study the semilocal convergence of the method. Most of them are modifications of the Newton-Kantorovich theorem in order to relax conditions $(i)-(i i i)$, specially condition $(i i)$. But if the condition required to the operator $F$ is milder than (ii), as we can see in $[4,5,8,9,18,20,21]$, then condition (iii) is usually replaced by other condition more restrictive, which necessarily leads to a reduction in the domain of valid starting points for Newton's method.

In this paper, our main aim is not to require milder conditions to the operator $F$, but stronger, which pursue a modification, not a restriction, of the valid starting points for Newton's method, so that this method can start at points from which the Newton-Kantorovich theorem cannot guarantee its semilocal convergence, as well as improving the domains of existence and uniqueness of solution and the a priori error estimates. For this, centered conditions $[3,13,22]$ are required to the operator $F^{\prime \prime}$. Like Kantorovich, our approach goes through to obtain a general semilocal convergence result by using the well-known majorant principle, that Kantorovich developed for Newton's method $[15,16]$. From this general result, we see other results as particular cases $[13,22]$.

The paper begins in Section 2 by recalling the concept of majorizing sequence and presenting the NewtonKantorovich theorem. In this section we also introduce the new convergence conditions for the general case. In Section 3, we present a new general semilocal convergence theorem for Newton's method and indicate how the majorizing sequences are constructed. We also include information about the existence and uniqueness of solution and a result on the a priori error estimates that leads to the quadratic convergence of Newton's method. In Section 4, we emphasize three particular cases of our general result that have been studied by other authors $($ see $[13,22])$. We finish the paper with two applications in Section 5, where nonlinear integral equations of mixed Hammerstein type are involved. We clearly show the advantages of our new semilocal convergence theorem with respect to the Newton-Kantorovich theorem.

Throughout the paper we denote $\overline{B(x, R)}=\{y \in X ;\|y-x\| \leq R\}$ and $B(x, R)=\{y \in X ;\|y-x\|<R\}$.

\section{BASELINE INFORMATION}

The famous Newton-Kantorovich theorem [16] guarantees the semilocal convergence of Newton's method in Banach spaces and gives a priori error estimates and information about the existence and uniqueness of solution. Kantorovich proves the theorem by using two different techniques [14,15], although the most prominent one is the majorant principle [15], which is based on the concept of majorizing sequence. This technique has been usually used later by other authors to analyse the semilocal convergence of several iterative methods $[1,2,5,21]$. We begin by introducing the concept of majorizing sequence and remembering how it is used to prove the convergence of sequences in Banach spaces.

Definition 2.1. If $\left\{x_{n}\right\}$ is a sequence in a Banach space $X$ and $\left\{t_{n}\right\}$ is a scalar sequence, then $\left\{t_{n}\right\}$ is a majorizing sequence of $\left\{x_{n}\right\}$ if $\left\|x_{n}-x_{n-1}\right\| \leq t_{n}-t_{n-1}$, for all $n \in \mathbb{N}$.

Observe, from the last inequality, it follows the sequence $\left\{t_{n}\right\}$ is non-decreasing. The interest of the majorizing sequence is that the convergence of the sequence $\left\{x_{n}\right\}$ in $X$ is deduced from the convergence of the scalar sequence $\left\{t_{n}\right\}$, as we can see in the following result [16]:

Lemma 2.2. Let $\left\{x_{n}\right\}$ be a sequence in a Banach space $X$ and $\left\{t_{n}\right\}$ a majorizing sequence of $\left\{x_{n}\right\}$. Then, if $\left\{t_{n}\right\}$ converges to $t^{*}<\infty$, there exists $x^{*} \in X$ such that $x^{*}=\lim _{n} x_{n}$ and $\left\|x^{*}-x_{n}\right\| \leq t^{*}-t_{n}$, for $n=0,1,2, \ldots$

From the last results, Kantorovich proves his well-known Newton-Kantorovich theorem, which is formulated as follows. 
Theorem 2.3 (the Newton-Kantorovich theorem). Let $F: \Omega \subseteq X \rightarrow X$ be a twice continuously differentiable operator defined on a non-empty open convex domain $\Omega$ of a Banach space $X$ with values in a Banach space $Y$. Suppose that conditions (i)-(iii) hold and $\overline{B\left(x_{0}, s^{*}\right)} \subseteq \Omega$, where $s^{*}=\frac{1-\sqrt{1-2 k \beta \eta}}{k \beta}$. Then Newton's sequence, given by (1.1), converges to a solution $x^{*}$ of the equation $F(x)=0$, starting at $x_{0}$, and $x_{n}, x^{*} \in \overline{B\left(x_{0}, s^{*}\right)}$, for all $n=0,1,2, \ldots$ Moreover, if $k \beta \eta<\frac{1}{2}, x^{*}$ is the unique solution of $F(x)=0$ in $B\left(x_{0}, s^{* *}\right) \cap \Omega$, where $s^{* *}=\frac{1+\sqrt{1-2 k \beta \eta}}{k \beta}$, and if $k \beta \eta=\frac{1}{2}, x^{*}$ is unique in $\overline{B\left(x_{0}, s^{*}\right)}$. Furthermore,

$$
\left\|x_{n+1}-x_{n}\right\| \leq s_{n+1}-s_{n} \quad \text { and } \quad\left\|x^{*}-x_{n}\right\| \leq s^{*}-s_{n}, \quad \text { for all } 0,1,2, \ldots,
$$

where $s_{n}=s_{n-1}-\frac{p\left(s_{n-1}\right)}{p^{\prime}\left(s_{n-1}\right)}$, with $n \in \mathbb{N}, s_{0}=0$ and $p(s)=\frac{k}{2} t^{2}-\frac{t}{\beta}+\frac{\eta}{\beta}$.

As we have written in the introduction, condition (ii) limits the application of the Newton-Kantorovich theorem. In Section 5 we illustrate this with two examples. Our idea in this paper is to generalize the hypotheses of Kantorovich by modifying conditions (ii) and (iii) and, following Kantorovich's theory [16], construct a real function $f \in \mathcal{C}^{(2)}\left(\left[t_{0}, t^{\prime}\right]\right)$ with $t_{0}, t^{\prime} \in \mathbb{R}$ which satisfies:

$\left(A_{1}\right)$ there exists $\Gamma_{0}=\left[F^{\prime}\left(x_{0}\right)\right]^{-1}$, for some $x_{0} \in \Omega$, with $\left\|\Gamma_{0}\right\| \leq-\frac{1}{f^{\prime}\left(t_{0}\right)}$ and $\left\|\Gamma_{0} F\left(x_{0}\right)\right\| \leq-\frac{f\left(t_{0}\right)}{f^{\prime}\left(t_{0}\right)}$, and $\left\|F^{\prime \prime}\left(x_{0}\right)\right\| \leq f^{\prime \prime}\left(t_{0}\right) ;$

$\left(A_{2}\right)\left\|F^{\prime \prime}(x)-F^{\prime \prime}\left(x_{0}\right)\right\| \leq f^{\prime \prime}(t)-f^{\prime \prime}\left(t_{0}\right)$, for $\left\|x-x_{0}\right\| \leq t-t_{0}, x \in \Omega$ and $t \in\left[t_{0}, t^{\prime}\right]$.

\section{MAIN RESULTS}

We use the majorant principle to prove the semilocal convergence of Newton's method under general conditions $\left(A_{1}\right)-\left(A_{2}\right)$, as Kantorovich does in [16] for classic conditions $(i)-(i i i)$. For this, we construct a majorizing sequence $\left\{t_{n}\right\}$ of Newton's sequence $\left\{x_{n}\right\}$ in the Banach space $X$. To obtain the sequence $\left\{t_{n}\right\}$ we use a real function $f(t)$ defined in $\left[t_{0}, t^{\prime}\right] \subset \mathbb{R}$ as follows:

$$
t_{0} \text { given, } \quad t_{n}=N\left(t_{n-1}\right)=t_{n-1}-\frac{f\left(t_{n-1}\right)}{f^{\prime}\left(t_{n-1}\right)}, \quad n \in \mathbb{N} .
$$

To build the majorizing sequence $\left\{t_{n}\right\}$ from $f(t)$, it is well-known [16] that it is necessary that the function $f(t)$ has at least one zero $t^{*}$, such that $t^{*} \geq t_{0}$, and the sequence $\left\{t_{n}\right\}$ is increasing and convergent to $t^{*}$. When this happens, the semilocal convergence of Newton's sequence $\left\{x_{n}\right\}$ is guaranteed in the Banach space $X$ from the convergence of the sequence $\left\{t_{n}\right\}$ (see Lem. 2.2).

To see the above-mentioned, we first study the convergence of the scalar sequence $\left\{t_{n}\right\}$ given in (3.1). If conditions $\left(A_{1}\right)-\left(A_{2}\right)$ are satisfied and there exits a root $\alpha \in\left(t_{0}, t^{\prime}\right)$ of $f^{\prime}(t)=0$ such that $f(\alpha) \leq 0$, then the equation $f(t)=0$ has only one root $t^{*}$ in $\left(t_{0}, \alpha\right)$. Indeed, if $f(\alpha)<0$, as $f\left(t_{0}\right)>0$, then $f(t)$ has at least one zero $t^{*}$ in $\left(t_{0}, \alpha\right)$ by continuity. Besides, since $f^{\prime \prime}\left(t_{0}\right) \geq 0$, from $\left(A_{2}\right)$ it follows that $f^{\prime \prime}(t) \geq 0$ for $t \in\left(t_{0}, \alpha\right)$, so that $f^{\prime}(t)$ is increasing and $f^{\prime}(t)<0$ for $t \in\left(t_{0}, \alpha\right)$. Also, as $f^{\prime}\left(t_{0}\right)<0, f(t)$ is decreasing for $t \in\left[t_{0}, \alpha\right)$. In consequence, $t^{*}$ is the unique root of $f(t)=0$ in $\left(t_{0}, \alpha\right)$. On the other hand, if $f(\alpha)=0$, then $\alpha$ is a double root of $f(t)=0$ and we choose $t^{*}=\alpha$.

The convergence of the scalar sequence $\left\{t_{n}\right\}$ is guaranteed from the next theorem.

Theorem 3.1. Let $\left\{t_{n}\right\}$ be the scalar sequence given in (3.1) with $f \in \mathcal{C}^{(2)}\left(\left[t_{0}, t^{\prime}\right]\right)$. Suppose that conditions $\left(A_{1}\right)-\left(A_{2}\right)$ hold and there exists a root $\alpha \in\left(t_{0}, t^{\prime}\right)$ of $f^{\prime}(t)=0$ such that $f(\alpha) \leq 0$. Then, $\left\{t_{n}\right\}$ is a nondecreasing sequence that converges to $t^{*}$.

Proof. As $f\left(t_{0}\right)>0$, then $t_{0}-t^{*} \leq 0$. By the mean value theorem, we obtain

$$
t_{1}-t^{*}=N\left(t_{0}\right)-N\left(t^{*}\right)=N^{\prime}\left(\theta_{0}\right)\left(t_{0}-t^{*}\right) \quad \text { with } \quad \theta_{0} \in\left(t_{0}, t^{*}\right),
$$

so that $t_{1}<t^{*}$, since $N^{\prime}(t)=\frac{f(t) f^{\prime \prime}(t)}{f^{\prime}(t)^{2}}>0$ in $\left[t_{0}, t^{*}\right)$. 
On the other hand, we have

$$
t_{1}-t_{0}=-\frac{f\left(t_{0}\right)}{f^{\prime}\left(t_{0}\right)} \geq 0 .
$$

By mathematical induction on $n$, we obtain $t_{n}<t^{*}$ and $t_{n}-t_{n-1} \geq 0$, since $\left(t_{n-1}, t^{*}\right) \subset\left(t_{0}, t^{*}\right)$.

Therefore, we infer that sequence (3.1) converges to $r \in\left[t_{0}, t^{*}\right]$. Moreover, since $t^{*}$ is the unique root of $f(t)=0$ in $\left[t_{0}, t^{*}\right]$, it follows that $r=t^{*}$.

Before we see that (3.1) is a majorizing sequence of (1.1) in the Banach space $X$, we give the following technical lemma that is used later.

Lemma 3.2. Let $f \in \mathcal{C}^{(2)}\left(\left[t_{0}, t^{\prime}\right]\right)$ with $t_{0}, t^{\prime} \in \mathbb{R}$. Suppose that conditions $\left(A_{1}\right)-\left(A_{2}\right)$ hold and there exits $\alpha \in\left(t_{0}, t^{\prime}\right)$ such that $f^{\prime}(\alpha)=0$ and $B\left(x_{0}, \alpha-t_{0}\right) \subseteq \Omega$. Then, for $x \in B\left(x_{0}, \alpha-t_{0}\right)$, the operator $L_{F}(x)=$ $\Gamma F^{\prime \prime}(x) \Gamma F(x)$ exists, where $\Gamma=\left[F^{\prime}(x)\right]^{-1}$, and is such that

$$
\left\|L_{F}(x)\right\| \leq \frac{f^{\prime \prime}(t)}{f^{\prime}(t)^{2}}\|F(x)\| \quad \text { for } \quad\left\|x-x_{0}\right\| \leq t-t_{0} .
$$

Proof. We start proving that the operator $\Gamma$ exists for $x \in B\left(x_{0}, \alpha-t_{0}\right)$ and, for $t \in\left(t_{0}, \alpha\right)$ with $\left\|x-x_{0}\right\| \leq t-t_{0}$, we also have

$$
\left\|\Gamma F^{\prime}\left(x_{0}\right)\right\| \leq \frac{f^{\prime}\left(t_{0}\right)}{f^{\prime}(t)} \quad \text { and } \quad\|\Gamma\| \leq-\frac{1}{f^{\prime}(t)} .
$$

From

$$
\begin{aligned}
\left\|I-\Gamma_{0} F^{\prime}(x)\right\| & =\left\|-\Gamma_{0} \int_{x_{0}}^{x} F^{\prime \prime}(z) \mathrm{d} z\right\| \\
& \leq\left\|\Gamma_{0}\right\|\left\|F^{\prime \prime}\left(x_{0}\right)\right\|\left\|x-x_{0}\right\|+\left\|\Gamma_{0}\right\|\left\|\int_{x_{0}}^{x}\left(F^{\prime \prime}(z)-F^{\prime \prime}\left(x_{0}\right)\right) \mathrm{d} z\right\| \\
& \leq-\frac{f^{\prime \prime}\left(t_{0}\right)}{f^{\prime}\left(t_{0}\right)}\left(t-t_{0}\right)+\left\|\Gamma_{0}\right\|\left\|\int_{0}^{1}\left(F^{\prime \prime}\left(x_{0}+\tau\left(x-x_{0}\right)\right)-F^{\prime \prime}\left(x_{0}\right)\right)\left(x-x_{0}\right) \mathrm{d} \tau\right\|,
\end{aligned}
$$

and

$$
\left\|z-x_{0}\right\|=\tau\left\|x-x_{0}\right\| \leq \tau\left(t-t_{0}\right)=t_{0}+\tau\left(t-t_{0}\right)-t_{0}=u-t_{0},
$$

where $z=x_{0}+\tau\left(x-x_{0}\right)$ and $u=t_{0}+\tau\left(t-t_{0}\right)$, with $\tau \in[0,1]$, it follows from $\left(A_{2}\right)$ that

$$
\left\|I-\Gamma_{0} F^{\prime}(x)\right\| \leq-\frac{f^{\prime \prime}\left(t_{0}\right)}{f^{\prime}\left(t_{0}\right)}\left(t-t_{0}\right)-\frac{1}{f^{\prime}\left(t_{0}\right)} \int_{t_{0}}^{t}\left(f^{\prime \prime}(u)-f^{\prime \prime}\left(t_{0}\right)\right) \mathrm{d} u=1-\frac{f^{\prime}(t)}{f^{\prime}\left(t_{0}\right)}<1,
$$

since $f^{\prime}(t)$ is increasing and $f^{\prime}\left(t_{0}\right) \leq f^{\prime}(t) \leq 0$. In consequence, by the Banach lemma, the operator $\Gamma$ exists and

$$
\|\Gamma\| \leq \frac{\left\|\Gamma_{0}\right\|}{1-\left\|I-\Gamma_{0} F^{\prime}(x)\right\|} \leq-\frac{1}{f^{\prime}(t)}, \quad\left\|\Gamma F^{\prime}\left(x_{0}\right)\right\| \leq \frac{1}{1-\left\|I-\Gamma_{0} F^{\prime}(x)\right\|} \leq \frac{f^{\prime}\left(t_{0}\right)}{f^{\prime}(t)} .
$$

Therefore (3.3) holds.

On the other hand, if $x \in B\left(x_{0}, \alpha-t_{0}\right)$ and $t \in\left(t_{0}, \alpha\right)$ are such that $\left\|x-x_{0}\right\| \leq t-t_{0}$, we have

$$
\left\|F^{\prime \prime}(x)\right\| \leq\left\|F^{\prime \prime}\left(x_{0}\right)\right\|+\left\|F^{\prime \prime}(x)-F^{\prime \prime}\left(x_{0}\right)\right\| \leq f^{\prime \prime}\left(t_{0}\right)+f^{\prime \prime}(t)-f^{\prime \prime}\left(t_{0}\right)=f^{\prime \prime}(t)
$$

and (3.2) also holds.

Next, from the following lemma, we see that (3.1) is a majorizing sequence of sequence (1.1) in the Banach space $X$. 
Lemma 3.3. Under the hypotheses of Lemma 3.2, the following items are true for all $n=0,1,2, \ldots$

$\left(i_{n}\right) \quad x_{n} \in B\left(x_{0}, t^{*}-t_{0}\right) ;$

$\left(i i_{n}\right) \quad\left\|\Gamma_{0} F\left(x_{n}\right)\right\| \leq-\frac{f\left(t_{n}\right)}{f^{\prime}\left(t_{0}\right)}$

$\left(i i i_{n}\right)\left\|x_{n+1}-x_{n}\right\| \leq t_{n+1}-t_{n}$.

Proof. We prove $\left(i_{n}\right)-\left(i i i_{n}\right)$ by mathematical induction on $n$. Firstly, $x_{0}$ given, it is clear that $x_{1}$ is well-defined and

$$
\left\|x_{1}-x_{0}\right\|=\left\|\Gamma_{0} F\left(x_{0}\right)\right\| \leq-\frac{f\left(t_{0}\right)}{f^{\prime}\left(t_{0}\right)}=t_{1}-t_{0}<t^{*}-t_{0}
$$

Then $\left(i_{0}\right)-\left(i i i_{0}\right)$ hold.

We now suppose that $\left(i_{k}\right)-\left(i i_{k}\right)$ are true for $k=0,1, \ldots, n-1$ and prove that they are also true for $k=n$.

As $x_{n}=x_{n-1}-\left[F^{\prime}\left(x_{n-1}\right)\right]^{-1} F\left(x_{n-1}\right)$, it is clear that $x_{n}$ is well-defined, since $\left[F^{\prime}\left(x_{n-1}\right)\right]^{-1}$ exists by the last lemma. Moreover,

$$
\begin{aligned}
\left\|x_{n}-x_{0}\right\| & \leq\left\|x_{n}-x_{n-1}\right\|+\left\|x_{n-1}-x_{n-2}\right\|+\cdots+\left\|x_{1}-x_{0}\right\| \\
& \leq t_{n}-t_{n-1}+t_{n-1}-t_{n-2}+\cdots+t_{1}-t_{0}=t_{n}-t_{0}<t^{*}-t_{0},
\end{aligned}
$$

so that $x_{n} \in B\left(x_{0}, t^{*}-t_{0}\right)$ and $\left(i_{n}\right)$ holds.

After that, we consider $x=x_{n-1}+s\left(x_{n}-x_{n-1}\right)$, with $s \in[0,1]$, and $\left\|x-x_{n-1}\right\|=s\left\|x_{n}-x_{n-1}\right\| \leq s\left(t_{n}-t_{n-1}\right)$. Therefore,

$$
\begin{aligned}
\left\|x-x_{0}\right\| & \leq\left\|x_{n-1}-x_{0}\right\|+s\left\|x_{n}-x_{n-1}\right\| \leq t_{n-1}-t_{0}+s\left(t_{n}-t_{n-1}\right) \\
& =t_{n-1}+s\left(t_{n}-t_{n-1}\right)-t_{0}=t-t_{0},
\end{aligned}
$$

with $t=t_{n-1}+s\left(t_{n}-t_{n-1}\right) \in\left[t_{n-1}, t_{n}\right]$, and since $\left\|x-x_{0}\right\| \leq t-t_{0}<t^{*}-t_{0}$, it is clear that $x \in B\left(x_{0}, t^{*}-t_{0}\right)$ for $x \in\left[x_{n-1}, x_{n}\right]$. From Lemma 3.2, we have that the operators $\Gamma$ and $L_{F}(x)$ exist and

$$
\left\|L_{F}(x)\right\| \leq \frac{f^{\prime \prime}(t)}{f^{\prime}(t)^{2}}\|F(x)\| \quad \text { with } \quad t=t_{n-1}+s\left(t_{n}-t_{n-1}\right) \quad \text { and } \quad s \in[0,1] .
$$

Besides,

$$
\left\|L_{F}(x)\right\| \leq-\frac{f^{\prime \prime}(t)}{f^{\prime}(t)} \frac{f^{\prime}\left(t_{0}\right)}{f^{\prime}(t)}\left\|\Gamma_{0} F(x)\right\|,
$$

simply by writing $L_{F}(x)=\Gamma F^{\prime \prime}(x) \Gamma F^{\prime}\left(x_{0}\right) \Gamma_{0} F(x)$ and applying (3.3).

Taking now into account the last inequality and Taylor's series, we write

$$
\begin{aligned}
\left\|\Gamma_{0} F(x)\right\|= & \left\|\Gamma_{0} F\left(x_{n-1}\right)+\Gamma_{0} F^{\prime}\left(x_{n-1}\right)\left(x-x_{n-1}\right)+\int_{x_{n-1}}^{x} \Gamma_{0} F^{\prime \prime}(z)(x-z) \mathrm{d} z\right\| \\
\leq & (1-s)\left\|\Gamma_{0} F\left(x_{n-1}\right)\right\|+\frac{1}{2}\left\|\Gamma_{0}\right\|\left\|F^{\prime \prime}\left(x_{0}\right)\right\|\left\|x-x_{n-1}\right\|^{2} \\
& +\left\|\Gamma_{0}\right\| \int_{0}^{1}\left\|F^{\prime \prime}\left(x_{n-1}+\tau\left(x-x_{n-1}\right)\right)-F^{\prime \prime}\left(x_{0}\right)\right\|\left\|x-x_{n-1}\right\|^{2}(1-\tau) \mathrm{d} \tau .
\end{aligned}
$$


As $\left\|x-x_{n-1}\right\|=s\left\|x_{n}-x_{n-1}\right\| \leq s\left(t_{n}-t_{n-1}\right) \leq t-t_{n-1}$, for $z=x_{n-1}+\tau\left(x-x_{n-1}\right)$ with $\tau \in[0,1]$, we have $\left\|z-x_{0}\right\| \leq u-t_{0}$, where $u=t_{n-1}+\tau\left(t-t_{n-1}\right)$. Consequently,

$$
\begin{aligned}
\left\|\Gamma_{0} F(x)\right\| \leq & -(1-s) \frac{f\left(t_{n-1}\right)}{f^{\prime}\left(t_{0}\right)}-\frac{1}{2} \frac{f^{\prime \prime}\left(t_{0}\right)}{f^{\prime}\left(t_{0}\right)}\left(t-t_{n-1}\right)^{2} \\
& -\frac{1}{f^{\prime}\left(t_{0}\right)} \int_{0}^{1}\left(f^{\prime \prime}\left(t_{n-1}+\tau\left(t-t_{n-1}\right)\right)-f^{\prime \prime}\left(t_{0}\right)\right)\left(t_{n}-t_{n-1}\right)^{2}(1-\tau) \mathrm{d} \tau \\
= & -\frac{1}{f^{\prime}\left(t_{0}\right)}\left(f\left(t_{n-1}\right)+f^{\prime}\left(t_{n-1}\right)\left(t-t_{n-1}\right)+\int_{t_{n-1}}^{t} f^{\prime \prime}(u)(t-u) \mathrm{d} u\right) \\
= & -\frac{f(t)}{f^{\prime}\left(t_{0}\right)}
\end{aligned}
$$

If we take $s=1$ above, we obtain $x=x_{n}, t=t_{n}$ and $\left\|\Gamma_{0} F\left(x_{n}\right)\right\| \leq-\frac{f\left(t_{n}\right)}{f^{\prime}\left(t_{0}\right)}$. In addition, $\left(i i_{n}\right)$ holds and $\left\|L_{F}(x)\right\| \leq \frac{f(t) f^{\prime \prime}(t)}{f^{\prime}(t)^{2}}=L_{f}(t)$.

Finally, to prove $\left(i i_{n}\right)$, just see that

$$
\begin{aligned}
\left\|x_{n+1}-x_{n}\right\| & =\left\|\int_{x_{n-1}}^{x_{n}} L_{F}(x) \mathrm{d} x\right\| \\
& \leq \int_{0}^{1}\left\|L_{F}\left(x_{n-1}+\tau\left(x_{n}-x_{n-1}\right)\right)\right\|\left\|x_{n}-x_{n-1}\right\| \mathrm{d} \tau \\
& \leq \int_{0}^{1} L_{f}\left(t_{n-1}+\tau\left(t_{n}-t_{n-1}\right)\right)\left(t_{n}-t_{n-1}\right) \mathrm{d} \tau \\
& =\int_{t_{n-1}}^{t_{n}} L_{f}(u) \mathrm{d} u \\
& =t_{n+1}-t_{n},
\end{aligned}
$$

since $x=x_{n-1}+\tau\left(x_{n}-x_{n-1}\right)$ with $\tau \in[0,1]$ and

$$
\left\|x-x_{0}\right\| \leq\left\|x_{n-1}-x_{0}\right\|+\tau\left\|x_{n}-x_{n-1}\right\| \leq t_{n-1}+\tau\left(t_{n}-t_{n-1}\right)-t_{0}=t-t_{0},
$$

where $t=t_{n-1}+\tau\left(t_{n}-t_{n-1}\right)$.

Once we have seen that (3.1) is a majorizing sequence of (1.1), we are ready to prove the semilocal convergence of (1.1) in the Banach space $X$.

Theorem 3.4 (the semilocal convergence theorem). Let $X$ and $Y$ be two Banach spaces and $F: \Omega \subseteq X \rightarrow Y$ a nonlinear twice continuously differentiable operator on a non-empty open convex domain $\Omega$ and $f \in \overline{\mathcal{C}}^{(2)}\left(\left[t_{0}, t^{\prime}\right]\right)$ with $t_{0}, t^{\prime} \in \mathbb{R}$. Suppose that $\left(A_{1}\right)-\left(A_{2}\right)$ hold, there exists a root $\alpha \in\left(t_{0}, t^{\prime}\right)$ of $f^{\prime}(t)=0$ such that $f(\alpha) \leq 0$, and $\overline{B\left(x_{0}, t^{*}-t_{0}\right)} \subseteq \Omega$. Then, Newton's sequence $\left\{x_{n}\right\}$, given by (1.1), converges to a solution $x^{*}$ of $F(x)=0$ starting at $x_{0}$. Moreover, $x_{n}, x^{*} \in \overline{B\left(x_{0}, t^{*}-t_{0}\right)}$ and

$$
\left\|x^{*}-x_{n}\right\| \leq t^{*}-t_{n}, \quad \text { for all } n=0,1,2, \ldots,
$$

where $\left\{t_{n}\right\}$ is defined in (3.1). 
Proof. Observe that $\left\{x_{n}\right\}$ is convergent, since $\left\{t_{n}\right\}$ is a majorizing sequence of $\left\{x_{n}\right\}$ and convergent. Moreover, as $\lim _{n \rightarrow+\infty} t_{n}=t^{*}$, if $x^{*}=\lim _{n \rightarrow+\infty} x_{n}$, then $\left\|x^{*}-x_{n}\right\| \leq t^{*}-t_{n}$, for all $n=0,1,2, \ldots$ Furthermore, from item $\left(i i_{n}\right)$ of the last lemma, we have $\left\|\Gamma_{0} F\left(x_{n}\right)\right\| \leq-\frac{f\left(t_{n}\right)}{f^{\prime}\left(t_{0}\right)}$, for all $n=0,1,2, \ldots$ Then, by letting $n \rightarrow+\infty$ in the last inequality, it follows $F\left(x^{*}\right)=0$ by the continuity of $F$.

After proving the semilocal convergence of Newton's method and locating the solution $x^{*}$, we prove the uniqueness of $x^{*}$. Note that if $f(t)$ has two real zeros $t^{*}$ and $t^{* *}$ such that $t_{0}<t^{*} \leq t^{* *}$, then the uniqueness of solution follows from the next theorem.

Theorem 3.5 (uniqueness of solution). Under the hypotheses of Theorem 3.4, the solution $x^{*}$ is unique in $B\left(x_{0}, t^{* *}-t_{0}\right) \cap \Omega$ if $t^{*}<t^{* *}$ or in $\overline{B\left(x_{0}, t^{* *}-t_{0}\right)}$ if $t^{*}=t^{* *}$.

Proof. Suppose that $t^{*}<t^{* *}$ and $y^{*}$ is another solution of $F(x)=0$ in $B\left(x_{0}, t^{* *}-t_{0}\right) \cap \Omega$. Then,

$$
\left\|y^{*}-x_{0}\right\| \leq \rho\left(t^{* *}-t_{0}\right) \quad \text { with } \rho \in(0,1) .
$$

We now suppose that $\left\|y^{*}-x_{k}\right\| \leq \rho^{2^{k}}\left(t^{* *}-t_{k}\right)$ for $k=0,1, \ldots, n$. In addition,

$$
\begin{aligned}
\left\|y^{*}-x_{n+1}\right\|= & \left\|-\Gamma_{n}\left(F\left(y^{*}\right)-F\left(x_{n}\right)-F^{\prime}\left(x_{n}\right)\left(y^{*}-x_{n}\right)\right)\right\| \\
= & \|-\Gamma_{n}\left(\int_{0}^{1}\left(F^{\prime \prime}\left(x_{n}+\tau\left(y^{*}-x_{n}\right)\right)-F^{\prime \prime}\left(x_{0}\right)\right)(1-\tau)\left(y^{*}-x_{n}\right)^{2} \mathrm{~d} \tau\right. \\
& \left.+\frac{1}{2} F^{\prime \prime}\left(x_{0}\right)\left(y^{*}-x_{n}\right)^{2}\right) \| .
\end{aligned}
$$

As $\left\|x_{n}+\tau\left(y^{*}-x_{n}\right)-x_{0}\right\| \leq t_{n}+\tau\left(t^{* *}-t_{n}\right)-t_{0}$, it follows that

$$
\left\|y^{*}-x_{n+1}\right\| \leq-\frac{\mu}{f^{\prime}\left(t_{n}\right)}\left\|y^{*}-x_{n}\right\|^{2}
$$

where $\mu=\frac{1}{2} f^{\prime \prime}\left(t_{0}\right)+\int_{0}^{1}\left(f^{\prime \prime}\left(t_{n}+\tau\left(t^{* *}-t_{n}\right)\right)-f^{\prime \prime}\left(t_{0}\right)\right)(1-\tau) \mathrm{d} \tau$.

On the other hand, we also have

$$
\begin{aligned}
t^{* *}-t_{n+1} & =-\frac{1}{f^{\prime}\left(t_{n}\right)}\left(\int_{t_{n}}^{t^{* *}}\left(f^{\prime \prime}(t)-f^{\prime \prime}\left(t_{0}\right)\right)\left(t^{* *}-t\right) \mathrm{d} t+\frac{1}{2} f^{\prime \prime}\left(t_{0}\right)\left(t^{* *}-t_{n}\right)^{2}\right) \\
& =-\frac{\mu}{f^{\prime}\left(t_{n}\right)}\left(t^{* *}-t_{n}\right)^{2} .
\end{aligned}
$$

Therefore,

$$
\left\|y^{*}-x_{n+1}\right\| \leq \frac{t^{* *}-t_{n+1}}{\left(t^{* *}-t_{n}\right)^{2}}\left\|y^{*}-x_{n}\right\|^{2} \leq \rho^{2^{n+1}}\left(t^{* *}-t_{n+1}\right)
$$

so that $y^{*}=x^{*}$.

If $t^{* *}=t^{*}$ and $y^{*}$ is another solution of $F(x)=0$ in $\overline{B\left(x_{0}, t^{* *}-t_{0}\right)}$, then $\left\|y^{*}-x_{0}\right\| \leq t^{*}-t_{0}$. Proceeding similarly to the previous case, we can prove by mathematical induction on $n$ that $\left\|y^{*}-x_{n}\right\| \leq t^{* *}-t_{n}$. Since $t^{* *}=t^{*}$ and $\lim _{n \rightarrow+\infty} t_{n}=t^{*}$, the uniqueness of solution is now easy to follow.

We finish this section by seeing the quadratic convergence of Newton's method under conditions $\left(A_{1}\right)-\left(A_{2}\right)$. We obtain the following theorem from Ostrsowski's technique [17]. The proof of the theorem can be found in [11].

Notice first that if $f(t)$ has two real zeros $t^{*}$ and $t^{* *}$ such that $t_{0}<t^{*} \leq t^{* *}$, we can then write

$$
f(t)=\left(t^{*}-t\right)\left(t^{* *}-t\right) g(t)
$$

with $g\left(t^{*}\right) \neq 0$ and $g\left(t^{* *}\right) \neq 0$. 
Theorem 3.6 (quadratic convergence of Newton's method). Let $f \in \mathcal{C}^{(2)}\left(\left[t_{0}, t^{\prime}\right]\right)$ with $t_{0}, t^{\prime} \in \mathbb{R}$. Suppose that conditions $\left(A_{1}\right)-\left(A_{2}\right)$ are satisfied and $f(t)$ has two real zeros $t^{*}$ and $t^{* *}$ such that $t_{0}<t^{*} \leq t^{* *}$.

(a) If $t^{*}<t^{* *}$, then

$$
\frac{\left(t^{* *}-t^{*}\right) \theta^{2^{n}}}{\sqrt{m_{1}}-\theta^{2^{n}}}<t^{*}-t_{n}<\frac{\left(t^{* *}-t^{*}\right) \Delta^{2^{n}}}{\sqrt{M_{1}}-\Delta^{2^{n}}}, \quad n \geq 0,
$$

where $\quad \theta=\frac{t^{*}}{t^{* *}} \sqrt{m_{1}}, \quad \Delta=\frac{t^{*}}{t^{* *}} \sqrt{M_{1}}, \quad m_{1}=\min \left\{H_{1}(t) ; t \in\left[0, t^{*}\right]\right\}, \quad M_{1}=\max \left\{H_{1}(t) ; t \in\left[0, t^{*}\right]\right\}$, $H_{1}(t)=\frac{\left(t^{* *}-t\right) g^{\prime}(t)-g(t)}{\left(t^{*}-t\right) g^{\prime}(t)-g(t)}$ and provided that $\theta<1$ and $\Delta<1$.

(b) If $t^{*}=t^{* *}$, then

$$
m_{2}^{n} t^{*} \leq t^{*}-t_{n} \leq M_{2}^{n} t^{*}
$$

where $m_{2}=\min \left\{H_{2}(t) ; t \in\left[0, t^{*}\right]\right\}, M_{2}=\max \left\{H_{2}(t) ; t \in\left[0, t^{*}\right]\right\}, H_{2}(t)=\frac{\left(t^{*}-t\right) g^{\prime}(t)-g(t)}{\left(t^{*}-t\right) g^{\prime}(t)-2 g(t)}$ and provided that $m_{2}<1$ and $M_{2}<1$.

From the last theorem, it follows that the convergence of Newton's method is quadratic if $t^{*}<t^{* *}$ and linear if $t^{*}=t^{* *}$.

\section{Particular cases}

To obtain the real function $f(t)$ from which the majorizing sequence $\left\{t_{n}\right\}$ is defined, Kantorovich considers that $f(t)$ is a second degree polynomial and fits its coefficients with conditions $(i)$ and $(i i)$. Kantorovich then obtains the polynomial

$$
p(s)=\frac{k}{2}\left(s-s_{0}\right)^{2}-\frac{s-s_{0}}{\beta}+\frac{\eta}{\beta} .
$$

Observe that this problem is of interpolation fitting.

In our case, if we consider $\left(A_{1}\right)-\left(A_{2}\right)$, we cannot obtain a real function by interpolation fitting, since $\left(A_{2}\right)$ does not allow determining the class of functions where $\left(A_{1}\right)$ can be applied. To solve this problem, we proceed differently. Observe that polynomial (4.1) can be obtained otherwise, without interpolation fitting, by solving the following initial value problem:

$$
\left\{\begin{array}{l}
p^{\prime \prime}(s)=k, \\
p\left(s_{0}\right)=\frac{\eta}{\beta}, \quad p^{\prime}\left(s_{0}\right)=-\frac{1}{\beta} .
\end{array}\right.
$$

Note that Kantorovich's polynomial (4.1) is such that

$$
p\left(s+s_{0}\right)=\bar{p}(s), \quad \text { where } \quad \bar{p}(s)=\frac{k}{2} s^{2}-\frac{s}{\beta}+\frac{\eta}{\beta} .
$$

Therefore, the scalar sequences given by Newton's method with $p(s)$ and $\bar{p}(s)$ can be obtained, one from the other, by translation. In consequence, the last results are independent of the value $s_{0}$. For this reason, we always choose $s_{0}=0$, which simplifies considerably the expressions used.

This new way of getting polynomial (4.1) has the advantage of being able to be generalized to conditions $\left(A_{1}\right)-$ $\left(A_{2}\right)$, so that we can then construct the real function $f$ under the general conditions considered in this paper. In the following, we see three different cases (already known) that can be deduced as particular cases of our general semilocal convergence theorem (Thm. 3.4). 


\section{1. $F^{\prime \prime}$ is centered Lipschitz}

Firstly, we suppose that conditions $\left(A_{1}\right)-\left(A_{2}\right)$ are reduced respectively to the following conditions:

$\left(\widetilde{A_{1}}\right)\left\|\Gamma_{0}\right\| \leq \beta,\left\|\Gamma_{0} F\left(x_{0}\right)\right\| \leq \eta,\left\|F^{\prime \prime}\left(x_{0}\right)\right\| \leq M$;

$\left(\widetilde{A_{2}}\right)\left\|F^{\prime \prime}(x)-F^{\prime \prime}\left(x_{0}\right)\right\| \leq k\left\|x-x_{0}\right\|$ for $x \in \Omega$.

Observe that condition $\left(\widetilde{A_{2}}\right)$ implicates condition $(i i)$ of Kantorovich in a bounded domain. On the other hand, to find a real function from conditions $\left(\widetilde{A_{1}}\right)-\left(\widetilde{A_{2}}\right)$, it is enough to solve the following initial value problem:

$$
\left\{\begin{array}{l}
\zeta^{\prime \prime}(t)=M+k\left(t-t_{0}\right), \\
\zeta\left(t_{0}\right)=\frac{\eta}{\beta}, \quad \zeta^{\prime}\left(t_{0}\right)=-\frac{1}{\beta},
\end{array}\right.
$$

whose solution is the cubic polynomial

$$
\zeta(t)=\frac{k}{6}\left(t-t_{0}\right)^{3}+\frac{M}{2}\left(t-t_{0}\right)^{2}-\frac{t-t_{0}}{\beta}+\frac{\eta}{\beta} .
$$

Note that polynomial (4.2) is such that

$$
\zeta\left(t+t_{0}\right)=\bar{\zeta}(t), \quad \text { where } \quad \bar{\zeta}(t)=\frac{k}{6} t^{3}+\frac{M}{2} t^{2}-\frac{t}{\beta}+\frac{\eta}{\beta} .
$$

Then, as for Kantorovich's polynomial (4.1), we choose $t_{0}=0$.

Notice that polynomial (4.2) satisfies the hypotheses of Theorem 3.4 and, consequently, the semilocal convergence of Newton's method is guaranteed in the Banach space $X$. In particular, from Theorem 3.4, we deduce the following semilocal convergence theorem.

Theorem 4.1. Let $X$ and $Y$ be two Banach spaces and $F: \Omega \subseteq X \rightarrow Y$ a nonlinear twice continuously differentiable operator on a non-empty open convex domain $\Omega$ and $\zeta(t)$ be the polynomial defined in (4.2) with $t_{0}=0$. Suppose that $\left(\widetilde{A_{1}}\right)-\left(\widetilde{A_{2}}\right)$ hold, $\zeta(\alpha) \leq 0$, where $\alpha=\frac{2}{M \beta+\sqrt{\beta\left(2 k+M^{2} \beta\right)}}$, and $\overline{B\left(x_{0}, t^{*}\right)} \subseteq \Omega$, where $t^{*}$ is the smallest positive root of $\zeta(t)=0$ with $t_{0}=0$. Then, Newton's sequence $\left\{x_{n}\right\}$, given by (1.1), converges to a solution $x^{*}$ of $F(x)=0$ starting at $x_{0}$. Moreover, $x_{n}, x^{*} \in \overline{B\left(x_{0}, t^{*}\right)}$ and

$$
\left\|x^{*}-x_{n}\right\| \leq t^{*}-t_{n}, \quad \text { for all } n=0,1,2, \ldots,
$$

where $t_{n}=t_{n-1}-\frac{\zeta\left(t_{n-1}\right)}{\zeta^{\prime}\left(t_{n-1}\right)}$, with $n \in \mathbb{N}, t_{0}=0$ and $\zeta(t)$ is defined in (4.2).

Observe that polynomial (4.2) with $t_{0}=0$ has a maximum at

$$
t=q=\frac{2}{M \beta-\sqrt{\beta\left(M^{2} \beta+2 k\right)}}<0
$$

and a minimum at

$$
t=\alpha=\frac{2}{M \beta+\sqrt{\beta\left(M^{2} \beta+2 k\right)}}>0 .
$$

Note also that if polynomial (4.2) with $t_{0}=0$ satisfies $\zeta(\alpha) \leq 0$, then (4.2) has a negative zero and two positive zeros $t^{*}$ and $t^{* *}$, such that $t^{*} \leq \alpha \leq t^{* *}$, and its graph is drawn in Figure 1 .

We also deduce from the previous study that the solution $x^{*}$ of $F(x)=0$ is unique in $B\left(x_{0}, t^{* *}\right) \cap \Omega$ if $t^{*}<t^{* *}$ or in $\overline{B\left(x_{0}, t^{*}\right)}$ if $t^{*}=t^{* *}$. Moreover, following Ostrowski's technique [17], we can prove some a priori error estimates that lead to the quadratic convergence of Newton's method when $t^{*}<t^{* *}$ or linear convergence when $t^{*}=t^{* *}$. These results, which are deduced from our study, can be found in [13]. Also, we remind that the condition $\zeta(\alpha) \leq 0$ can be replaced with equivalent or easier to prove, see [13] and the references given there. 


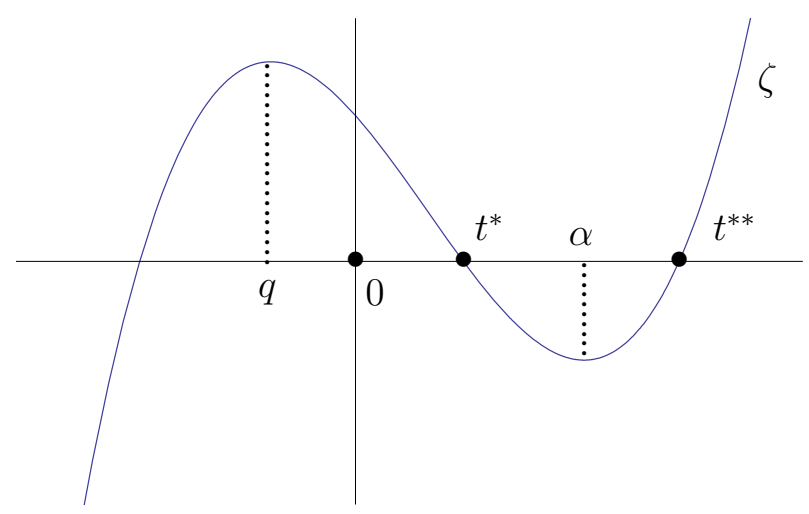

Figure 1. Graph of polynomial $\zeta(t)$ with $t_{0}=0$.

\section{2. $F^{\prime \prime}$ is centered Hölder}

Secondly, we suppose that conditions $\left(A_{1}\right)-\left(A_{2}\right)$ are reduced respectively to the following conditions

$\left(\overline{A_{1}}\right)\left\|\Gamma_{0}\right\| \leq \beta,\left\|\Gamma_{0} F\left(x_{0}\right)\right\| \leq \eta,\left\|F^{\prime \prime}\left(x_{0}\right)\right\| \leq M$;

$\left(\overline{A_{2}}\right)\left\|F^{\prime \prime}(x)-F^{\prime \prime}\left(x_{0}\right)\right\| \leq k\left\|x-x_{0}\right\|^{p}$, for $x \in \Omega, p \in[0,1]$.

Observe that condition $\left(\overline{A_{2}}\right)$ implicates condition $(i i)$ of Kantorovich in a bounded domain. Similarly to the previous case, to find a real function from conditions $\left(\overline{A_{1}}\right)-\left(\overline{A_{2}}\right)$, it is enough to solve the following initial value problem:

$$
\left\{\begin{array}{l}
\varphi^{\prime \prime}(t)=M+k\left(t-t_{0}\right)^{p}, \\
\varphi\left(t_{0}\right)=\frac{\eta}{\beta}, \quad \varphi^{\prime}\left(t_{0}\right)=-\frac{1}{\beta},
\end{array}\right.
$$

whose solution is the function

$$
\varphi(t)=\frac{k}{(p+1)(p+2)}\left(t-t_{0}\right)^{p+2}+\frac{M}{2}\left(t-t_{0}\right)^{2}-\frac{t-t_{0}}{\beta}+\frac{\eta}{\beta} .
$$

Observe that function (4.3) is reduced to polynomial (4.2) if $p=1$.

Note that function (4.3) is such that

$$
\varphi\left(t+t_{0}\right)=\bar{\varphi}(t), \quad \text { where } \quad \bar{\varphi}(t)=\frac{k}{(p+1)(p+2)} t^{p+2}+\frac{M}{2} t^{2}-\frac{t}{\beta}+\frac{\eta}{\beta} .
$$

Then, as for Kantorovich's polynomial (4.1), we choose $t_{0}=0$.

Notice also that function (4.3) satisfies the hypotheses of Theorem 3.4 and, consequently, the semilocal convergence of Newton's method is guaranteed in the Banach space $X$. In particular, from Theorem 3.4, we deduce the following semilocal convergence theorem.

Theorem 4.2. Let $X$ and $Y$ be two Banach spaces and $F: \Omega \subseteq X \rightarrow Y$ a nonlinear twice continuously differentiable operator on a non-empty open convex domain $\Omega$ and $\varphi(t)$ be the function defined in (4.3) with $t_{0}=0$. Suppose that $\left(\overline{A_{1}}\right)-\left(\overline{A_{2}}\right)$ hold, there exists a root $\alpha>0$ of $\varphi^{\prime}(t)=0$, such that $\varphi(\alpha) \leq 0$, and $\overline{B\left(x_{0}, t^{*}\right)} \subseteq$ $\Omega$, where $t^{*}$ is the smallest positive root of $\varphi(t)=0$ with $t_{0}=0$. Then, Newton's sequence $\left\{x_{n}\right\}$, given by (1.1), converges to a solution $x^{*}$ of $F(x)=0$ starting at $x_{0}$. Moreover, $x_{n}, x^{*} \in \overline{B\left(x_{0}, t^{*}\right)}$ and

$$
\left\|x^{*}-x_{n}\right\| \leq t^{*}-t_{n}, \quad \text { for all } n=0,1,2, \ldots,
$$

where $t_{n}=t_{n-1}-\frac{\varphi\left(t_{n-1}\right)}{\varphi^{\prime}\left(t_{n-1}\right)}$, with $n \in \mathbb{N}, t_{0}=0$ and $\varphi(t)$ is defined in (4.3). 
If we analyse the derivatives of $\varphi(t)$ with $t_{0}=0$, it is easy to see that the function $\varphi(t)$ has two positive real zeros $t^{*}$ and ${ }^{* *}$, such that $t^{*} \leq \alpha \leq t^{* *}$ if $\varphi(\alpha) \leq 0$, where $\alpha$ is the unique positive root of $\varphi^{\prime}(t)=0$.

We also deduce from the initial study that the solution $x^{*}$ of $F(x)=0$ is unique in $B\left(x_{0}, t^{* *}\right) \cap \Omega$ if $t^{*}<t^{* *}$ or in $\overline{B\left(x_{0}, t^{*}\right)}$ if $t^{*}=t^{* *}$. Moreover, following again Ostrowski's technique [17], we can prove some a priori error estimates that lead to the quadratic convergence of Newton's method when $t^{*}<t^{* *}$ or linear convergence when $t^{*}=t^{* *}$. These results, which are deduced from our study, also appear in [13].

\section{3. $F^{\prime \prime}$ is $\omega$-centered}

Thirdly, we suppose that conditons $\left(A_{1}\right)-\left(A_{2}\right)$ are reduced respectively to the following conditions:

$\left(\widehat{A_{1}}\right)\left\|\Gamma_{0}\right\| \leq \beta,\left\|\Gamma_{0} F\left(x_{0}\right)\right\| \leq \eta,\left\|F^{\prime \prime}\left(x_{0}\right)\right\| \leq M$;

$\left(\widehat{A_{2}}\right)\left\|F^{\prime \prime}(x)-F^{\prime \prime}\left(x_{0}\right)\right\| \leq \omega\left(\left\|x-x_{0}\right\|\right)$, where $\omega:[0,+\infty) \rightarrow \mathbb{R}$ is a non-decreasing continuos function such taht $\omega(0)=0$.

To find a real function from conditions $\left(\widehat{A_{1}}\right)-\left(\widehat{A_{2}}\right)$, it is enough to solve the following initial value problem:

$$
\left\{\begin{array}{l}
\phi^{\prime \prime}(t)=M+\omega\left(t-t_{0}\right), \\
\phi\left(t_{0}\right)=\frac{\eta}{\beta}, \quad \phi^{\prime}\left(t_{0}\right)=-\frac{1}{\beta},
\end{array}\right.
$$

whose solution is given in the following theorem.

Theorem 4.3. Suppose that the function $\omega\left(t-t_{0}\right)$ is continuous for all $t \in\left[t_{0}, t^{\prime}\right]$, with $t^{\prime}>t_{0}$. Then, for any real numbers $\beta \neq 0, \eta$ and $M$, there exists only one solution $\phi(t)$ of the last initial value problem in $\left[t_{0}, t^{\prime}\right]$; that is:

$$
\phi(t)=\int_{t_{0}}^{t} \int_{t_{0}}^{s} \omega\left(z-t_{0}\right) \mathrm{d} z \mathrm{~d} s+\frac{M}{2}\left(t-t_{0}\right)^{2}-\frac{t-t_{0}}{\beta}+\frac{\eta}{\beta}, \quad t_{0} \geq 0 .
$$

Observe that function (4.4) is reduced to functions (4.2) or (4.3) if $\omega(z)=k z$ or $\omega(z)=k z^{p}$, respectively.

To apply Theorem 3.4, the equation $\phi(t)=0$ must have at least one root greater than $t_{0}$, so that we have to guarantee the convergence of the scalar sequence $\left\{t_{n}\right\}$, from $t_{0}$, to this root. We then study the function $\phi(t)$ defined in (4.4).

Theorem 4.4. Let $\phi$ and $\omega$ be the functions defined respectively in (4.4) and $\left(\widehat{A_{2}}\right)$.

(a) If there exists a solution $\alpha>t_{0}$ of the equation

$$
\phi^{\prime}(t)=\int_{t_{0}}^{t} \omega\left(z-t_{0}\right) \mathrm{d} z+M\left(t-t_{0}\right)-\frac{1}{\beta}=0
$$

then $\alpha$ is the unique minimum of $\phi(t)$ in $\left[t_{0},+\infty\right)$ and $\phi(t)$ is non-increasing in $\left[t_{0}, \alpha\right)$.

(b) If $\phi(\alpha) \leq 0$, then the equation $\phi(t)=0$ has at least one root in $\left[t_{0},+\infty\right)$. Moreover, if $t^{*}$ is the smallest root of $\phi(t)=0$ in $\left[t_{0},+\infty\right)$, we have $t_{0}<t^{*} \leq \alpha$.

Taking into account the hypotheses of Theorem 4.4, function (4.4) satisfies the conditions of Theorem 3.4 and the semilocal convergence of Newton's method is then guaranteed in the Banach space $X$. In particular, we have the following theorem, whose proof follows immediately from Theorem 3.4.

Theorem 4.5. Let $X$ and $Y$ be two Banach spaces and $F: \Omega \subseteq X \rightarrow Y$ a nonlinear twice continuously differentiable operator on a non-empty open convex domain $\Omega$ and $\phi(t)$ be the function defined in (4.4) with $t_{0}=$ 0 . Suppose that $\left(\widehat{A_{1}}\right)-\left(\widehat{A_{2}}\right)$ hold, there exists a root $\alpha>t_{0}$ of $(4.5)$, such that $\phi(\alpha) \leq 0$, and $\overline{B\left(x_{0}, t^{*}-t_{0}\right)} \subseteq \Omega$, 
where $t^{*}$ is the smallest root of $\phi(t)=0$ in $\left[t_{0},+\infty\right)$. Then, Newton's sequence $\left\{x_{n}\right\}$, given by (1.1), converges to a solution $x^{*}$ of $F(x)=0$ starting at $x_{0}$. Moreover, $x_{n}, x^{*} \in \overline{B\left(x_{0}, t^{*}-t_{0}\right)}$ and

$$
\left\|x^{*}-x_{n}\right\| \leq t^{*}-t_{n}, \quad \text { for all } n=0,1,2, \ldots,
$$

where $t_{n}=t_{n-1}-\frac{\phi\left(t_{n-1}\right)}{\phi^{\prime}\left(t_{n-1}\right)}$, with $n \in \mathbb{N}$ and $\phi(t)$ is defined in (4.4).

Note that function (4.4) is such that

$$
\phi\left(t+t_{0}\right)=\bar{\phi}(t), \quad \text { where } \bar{\phi}(t)=\int_{0}^{t} \int_{0}^{s} \omega(z) \mathrm{d} z \mathrm{~d} s+\frac{M}{2} t^{2}-\frac{t}{\beta}+\frac{\eta}{\beta} .
$$

Then, as for Kantorovich's polynomial (4.1), we choose $t_{0}=0$.

\section{Applications}

As we have indicated in the introduction, the main aim of this paper is to require stronger conditions to the operator $F^{\prime \prime}$ than condition (ii), so that the domain of starting points for Newton's method is modified with respect to that of Kantorovich under conditions $(i)-(i i i)$. In the following, we see three different situations where Kantorovich's study is improved from conditions $\left(\widehat{A_{1}}\right)-\left(\widehat{A_{2}}\right)$ for one reason or another.

The three situations presented are particular cases of nonlinear integral equations of the following mixed Hammerstein type [12]:

$$
x(s)=g(s)+\int_{a}^{b} G(s, t) H(x(t)) \mathrm{d} t, \quad s \in[a, b],
$$

where $-\infty<a<b<+\infty, G, H$ and $g$ are known functions and $x$ is the function to determine. Integral equations of this type appear very often in several applications to real world problems. For example, in problems of dynamic models of chemical reactors [6], vehicular traffic theory, biology and queuing theory [7]. The Hammerstein integral equations also appear in the electro-magnetic fluid dynamics and can be reformulated as two-point boundary value problems with certain nonlinear boundary conditions and in multi-dimensional analogues which appear as reformulations of elliptic partial differentiable equations with nonlinear boundary conditions (see [19] and the references given there).

In particular, in this paper, we consider nonlinear equations of the form:

$$
x(s)=g(s)+\int_{a}^{b} G(s, t)\left(\lambda x(t)^{3}+\delta x(t)^{2+p}\right) \mathrm{d} t, \quad s \in[a, b],
$$

$p \in[0,1]$ and $\lambda, \delta \in \mathbb{R}$, where $g$ is a continuous function in $[a, b] \times[a, b]$ and the kernel $G$ is the Green function.

Solving equation (5.1) is equivalent to solve $F(x)=0$, where $F: \Omega \subset \mathcal{C}([a, b]) \rightarrow \mathcal{C}([a, b]), \Omega=\{x \in$ $\mathcal{C}([a, b]) ; x(s) \geq 0, s \in[a, b]\}$ and

$$
[F(x)](s)=x(s)-g(s)-\int_{a}^{b} G(s, t)\left(\lambda x(t)^{3}+\delta x(t)^{2+p}\right) \mathrm{d} t,
$$

where $s \in[a, b], p \in[0,1]$ and $\lambda, \delta \in \mathbb{R}$. In this case,

$$
\begin{gathered}
{\left[F^{\prime}(x) y\right](s)=y(s)-\int_{a}^{b} G(s, t)\left(3 \lambda x(t)^{2}+(2+p) \delta x(t)^{1+p}\right) y(t) \mathrm{d} t,} \\
{\left[F^{\prime \prime}(x)(y z)\right](s)=-\int_{a}^{b} G(s, t)\left(6 \lambda x(t)+(2+p)(1+p) \delta x(t)^{p}\right) z(t) y(t) \mathrm{d} t .}
\end{gathered}
$$


Notice that condition (ii) of Kantorovich is not satisfied, since $\left\|F^{\prime \prime}(x)\right\|$ is not bounded in $\Omega$. Moreover, it is not easy to locate a domain where $\left\|F^{\prime \prime}(x)\right\|$ is bounded and contains a solution of $F(x)=0$. Notice also that conditions $\left(\widetilde{A_{2}}\right)$ and $\left(\overline{A_{2}}\right)$ are not satisfied either, since $F^{\prime \prime}(x)$ is not Lipschitz-continuous or Hölder-continuous in $\Omega$, so that we cannot apply the results given in [13] to guarantee the semilocal convergence of Newton's method to a solution of (5.1).

Initially, we transform equation (5.1) into a finite dimensional problem and, later, we apply Newton's method to approximate solutions of (5.1). Then, we approximate the integral of (5.1) by a Gauss-Legendre quadrature formula with $m$ nodes:

$$
\int_{a}^{b} h(t) \mathrm{d} t \simeq \sum_{i=1}^{m} \varpi_{i} h\left(t_{i}\right)
$$

where the nodes $t_{i}$ and the weights $\varpi_{i}$ are determined. Now, if we denote the approximations of $x\left(t_{i}\right)$ and $g\left(t_{i}\right)$ by $x_{i}$ and $g_{i}$, respectively, with $i=1,2, \ldots, m$, then equation (5.1) is equivalent to the following nonlinear system of equations:

$$
x_{i}=g_{i}+\sum_{j=1}^{m} b_{i j}\left(\lambda x_{j}^{3}+\delta x_{j}^{2+p}\right), \quad i=1,2, \ldots, m,
$$

where

$$
b_{i j}= \begin{cases}\varpi_{j} \frac{\left(b-t_{i}\right)\left(t_{j}-a\right)}{b-a} & \text { if } j \leq i, \\ \varpi_{j} \frac{\left(b-t_{j}\right)\left(t_{i}-a\right)}{b-a} & \text { if } j>i .\end{cases}
$$

The last nonlinear system can be now written as follows:

$$
F(\bar{x})=\bar{x}-\bar{g}-B(\lambda \widetilde{x}+\delta \widehat{x})=0,
$$

where $\bar{x}=\left(x_{1}, x_{2}, \ldots, x_{m}\right)^{T}, \bar{g}=\left(g_{1}, g_{2}, \ldots, g_{m}\right)^{T}, B=\left(b_{i j}\right), \widetilde{x}=\left(x_{1}^{3}, x_{2}^{3}, \ldots, x_{m}^{3}\right)^{T}$ and $\widehat{x}=$ $\left(x_{1}^{2+p}, x_{2}^{2+p}, \ldots, x_{m}^{2+p}\right)^{T}$. In view of what the domain $\Omega$ is for equation (5.1), we consider $F: \Lambda \subset \mathbb{R}^{m} \rightarrow \mathbb{R}^{m}$, where $\Lambda=\left\{\left(x_{1}, x_{2}, \ldots, x_{m}\right) \in \mathbb{R}^{m} ; x_{i} \geq 0\right.$ for $\left.i=1,2, \ldots, m\right\}$. In addition,

$$
\begin{gathered}
F(\bar{x})=\left(\begin{array}{c}
x_{1}-g_{1}-\sum_{j=1}^{m} b_{1 j}\left(\lambda x_{i}^{3}+\delta x_{i}^{2+p}\right) \\
x_{2}-g_{2}-\sum_{j=1}^{m} b_{2 j}\left(\lambda x_{i}^{3}+\delta x_{i}^{2+p}\right) \\
\vdots \\
x_{m}-g_{m}-\sum_{j=1}^{m} b_{m j}\left(\lambda x_{i}^{3}+\delta x_{i}^{2+p}\right)
\end{array}\right), \\
F^{\prime}(\bar{x})=I-B\left(3 \lambda D_{1}(\bar{x})+(2+p) \delta D_{2}(\bar{x})\right),
\end{gathered}
$$

where $D_{1}(\bar{x})=\operatorname{diag}\left\{x_{1}^{2}, x_{2}^{2}, \ldots, x_{m}^{2}\right\}$ and $D_{2}(\bar{x})=\operatorname{diag}\left\{x_{1}^{1+p}, x_{2}^{1+p}, \ldots, x_{m}^{1+p}\right\}$, and

$$
F^{\prime \prime}(\bar{x}) \bar{y} \bar{z}=-B\left(\left(6 \lambda x_{1}+(2+p)(1+p) \delta x_{1}^{p}\right) y_{1} z_{1}, \ldots,\left(6 \lambda x_{m}+(2+p)(1+p) \delta x_{m}^{p}\right) y_{m} z_{m}\right)^{T},
$$

where $\bar{y}=\left(y_{1}, y_{2}, \ldots, y_{m}\right)^{T}$ and $\bar{z}=\left(z_{1}, z_{2}, \ldots, z_{m}\right)^{T}$.

Moreover, provided that $\|B\|\left(3|\lambda|\left\|D_{1}\left(\bar{x}_{0}\right)\right\|+(2+p)|\delta|\left\|D_{2}\left(\bar{x}_{0}\right)\right\|\right)<1$, we have

$$
\begin{gathered}
\left\|\Gamma_{0}\right\| \leq \frac{1}{1-\|B\|\left(3|\lambda||| D_{1}\left(\bar{x}_{0}\right)\|+(2+p)|\delta|\| D_{2}\left(\bar{x}_{0}\right) \|\right)}=\beta, \\
\left\|\Gamma_{0} F\left(\bar{x}_{0}\right)\right\| \leq \frac{\left\|\bar{x}_{0}-\bar{g}-B\left(|\lambda| \widetilde{x}_{0}+\delta \widetilde{x}_{0}\right)\right\|}{1-\|B\|\left(3 \lambda\left\|D_{1}\left(\bar{x}_{0}\right)\right\|+(2+p)|\delta|\left\|D_{2}\left(\bar{x}_{0}\right)\right\|\right)}=\eta,
\end{gathered}
$$


where $\bar{x}_{0}=\left(\check{x}_{1}, \check{x}_{2}, \ldots, \check{x}_{m}\right)^{T}, \widetilde{x}_{0}=\left(\check{x}_{1}^{3}, \check{x}_{2}^{3}, \ldots, \check{x}_{m}^{3}\right)^{T}$ and $\widehat{x}_{0}=\left(\check{x}_{1}^{2+p}, \check{x}_{2}^{2+p}, \ldots, \check{x}_{m}^{2+p}\right)^{T}$. Furthermore,

$$
\left\|F^{\prime \prime}(\bar{x})\right\|=\sup _{\|\bar{y}\|=1,\|\bar{z}\|=1}\left\|F^{\prime \prime}(\bar{x}) \overline{y z}\right\| \quad \text { with } \quad\left\|F^{\prime \prime}(\bar{x}) \overline{y z}\right\| \leq\|B\|\|v(\bar{x}, \bar{y}, \bar{z})\|,
$$

where $v(\bar{x}, \bar{y}, \bar{z})=\left(\left(6 \lambda x_{1}+(2+p)(1+p) \delta x_{1}^{p}\right) y_{1} z_{1}, \ldots,\left(6 \lambda x_{m}+(2+p)(1+p) \delta x_{m}^{p}\right) y_{m} z_{m}\right)^{T}$.

For the infinity norm, we have

$$
\|v(\bar{x}, \bar{y}, \bar{z})\|_{\infty}=\left(6|\lambda|\|\bar{x}\|_{\infty}+(2+p)(1+p)|\delta|\left\|x_{i}\right\|_{\infty}^{p}\right)\|\bar{y}\|_{\infty}\|\bar{z}\|_{\infty},
$$

so that

$$
\left\|F^{\prime \prime}(\bar{x})\right\|_{\infty} \leq\|B\|_{\infty}\left(6|\lambda|\|\bar{x}\|_{\infty}+(2+p)(1+p)|\delta|\|\bar{x}\|_{\infty}^{p}\right) .
$$

Consequently,

$$
\begin{gathered}
\left\|F^{\prime \prime}\left(\bar{x}_{0}\right)\right\|_{\infty} \leq\|B\|_{\infty}\left(6|\lambda|\left\|\bar{x}_{0}\right\|_{\infty}+(2+p)(1+p)|\delta|\left\|\bar{x}_{0}\right\|_{\infty}^{p}\right)=M, \\
\left\|F^{\prime \prime}(\bar{x})-F^{\prime \prime}\left(\bar{x}_{0}\right)\right\|_{\infty} \leq\|B\|_{\infty}\left(6|\lambda|\left\|\bar{x}-\bar{x}_{0}\right\|_{\infty}+(2+p)(1+p)|\delta|\left\|\bar{x}-\bar{x}_{0}\right\|_{\infty}^{p}\right)
\end{gathered}
$$

and then $\omega(z)=\|B\|_{\infty}\left(6|\lambda| z+(2+p)(1+p)|\delta| z^{p}\right)$.

Observe that in this case $\left\|F^{\prime \prime}(\bar{x})\right\|_{\infty}$ is not bounded in general, since the function $\chi(t)=6|\lambda| t+(2+p)(1+p)|\delta| t^{p}$ is increasing. Therefore, condition (ii) of Kantorovich is not satisfied.

\subsection{Application 1}

To solve the difficulty of applying Kantorovich's conditions, a common alternative is to locate the solutions in a domain $\Omega \subset \Lambda$ and look for a bound for $\left\|F^{\prime \prime}(\bar{x})\right\|_{\infty}$ in $\Omega$ (see [10]). In the next example we see that we cannot use this alternative either, because a priori we cannot find a domain $\Omega$ which contains solutions of the equation.

We consider the equation of type (5.1) given by

$$
x(s)=1+\int_{0}^{1} G(s, t)\left(x(t)^{3}+\frac{1}{4} x(t)^{\frac{5}{2}}\right) \mathrm{d} t, \quad s \in[0,1] .
$$

Once equation (5.1) is discretized, the solutions $\bar{x}^{*}$ of the corresponding nonlinear system given by (5.2) must satisfy

$$
\left\|\bar{x}^{*}\right\|_{\infty}-1-\|B\|\left(\left\|\bar{x}^{*}\right\|_{\infty}^{3}+\frac{1}{4}\left\|\bar{x}^{*}\right\|_{\infty}^{\frac{5}{2}}\right) \leq 0
$$

which does not imply restrictions on $\left\|\bar{x}^{*}\right\|_{\infty}$, so that we cannot locate a domain $\Omega \subset \Lambda$ where $\left\|F^{\prime \prime}(\bar{x})\right\|_{\infty}$ is bounded and contains $\bar{x}^{*}$. In consequence, we cannot guarantee the semilocal convergence of Newton's method to a discretized solution of (5.3) from the Newton-Kantorovich theorem.

However, we can do it from Theorem 4.5. If we choose $m=8, t_{0}=0$ and $\bar{x}_{0}=(1,1, \ldots, 1)^{T}$, we obtain $\beta=1.7248 \ldots, \eta=0.2499 \ldots, M=0.8571 \ldots, \alpha=0.5236 \ldots$,

$$
\phi(t)=(0.1449 \ldots)-(0.5797 \ldots) t+(0.4285 \ldots) t^{2}+(0.0308 \ldots) t^{\frac{5}{2}}+(0.1235 \ldots) t^{3}
$$

and $\phi(\alpha)=-0.0172 \ldots \leq 0$. In addition, after five iterations, Newton's method converges to the solution $\bar{x}^{*}=\left(x_{1}^{*}, x_{2}^{*}, \ldots, x_{8}^{*}\right)^{T}$ which is shown in Table 1 . Moreover, since $t^{*}=0.3596 \ldots$ and $t^{* *}=0.6822 \ldots$, the domains of existence and uniqueness of solution are respectively

$$
\left\{\bar{\nu} \in \Lambda ;\|\bar{\nu}-\overline{1}\|_{\infty} \leq 0.3596 \ldots\right\} \quad \text { and } \quad\left\{\bar{\nu} \in \Lambda ;\|\bar{\nu}-\overline{1}\|_{\infty}<0.6822 \ldots\right\} .
$$

If we interpolate the values given in Table 1 and take into account that equation (5.3) satisfies $x(0)=x(1)=1$, we obtain the solution drawn in Figure 2 and denoted by $\widetilde{x}$. 
TABLE 1. Numerical solution of (5.3).

\begin{tabular}{|c|c|c|c|}
\hline$i$ & $x_{i}^{*}$ & $i$ & $x_{i}^{*}$ \\
\hline 1 & $1.021626 \ldots$ & 5 & $1.302053 \ldots$ \\
2 & $1.105232 \ldots$ & 6 & $1.218581 \ldots$ \\
3 & $1.218581 \ldots$ & 7 & $1.105232 \ldots$ \\
4 & $1.302053 \ldots$ & 8 & $1.021626 \ldots$ \\
\hline
\end{tabular}

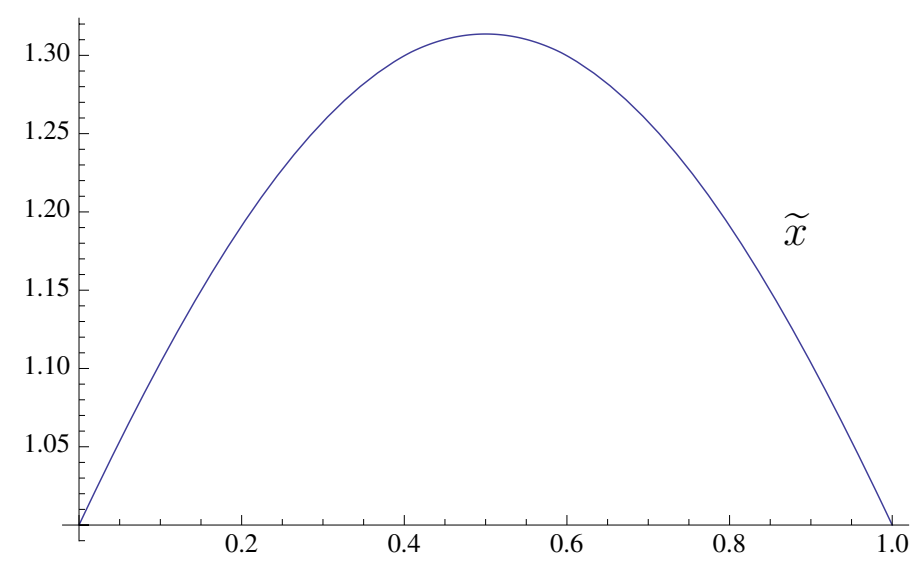

Figure 2. Approximated solution of equation (5.3).

\subsection{Application 2}

We now consider the following equation of type (5.1):

$$
x(s)=1+\int_{0}^{1} G(s, t)\left(x(t)^{3}+\frac{1}{5} x(t)^{\frac{5}{2}}\right) \mathrm{d} t, \quad s \in[0,1] .
$$

Once this equation is discretized with $m=8$, the solutions $\bar{x}^{*}$ of the corresponding nonlinear system given by (5.2) must satisfy

$$
\left\|\bar{x}^{*}\right\|_{\infty}-1-\|B\|_{\infty}\left(\left\|\bar{x}^{*}\right\|_{\infty}^{3}+\frac{1}{5}\left\|\bar{x}^{*}\right\|_{\infty}^{\frac{5}{2}}\right) \leq 0
$$

which is true provided that $\left\|\bar{x}^{*}\right\|_{\infty} \leq r_{1}=1.38003 \ldots$ or $\left\|\bar{x}^{*}\right\|_{\infty} \geq r_{2}=1.69922 \ldots$, where $r_{1}$ and $r_{2}$ are the two positive real roots of the scalar equation $t-1-\|B\|_{\infty}\left(t^{3}+\frac{1}{5} t^{\frac{5}{2}}\right)=0$, where $\|B\|_{\infty}=0.1235 \ldots$ Note that Newton's method can approximate solutions $\bar{x}^{*}$ such that $\left\|\bar{x}^{*}\right\|_{\infty} \in\left[0, r_{1}\right]$ if we apply the Newton-Kantorovich theorem, since we can consider $\Omega=B(0, r) \cap \Lambda$ with $r \in\left(r_{1}, r_{2}\right)$, where $\left\|F^{\prime \prime}(\bar{x})\right\|_{\infty}$ is bounded and choose a starting point $x_{0} \in \Omega$ such that condition (iii) is satisfied. However, the Newton-Kantorovich theorem cannot guarantee the convergence of Newton's method to solutions $\bar{x}^{* *}$ such that $\left\|\bar{x}^{* *}\right\|_{\infty} \geq r_{2}$, because we cannot choose a domain where $\bar{x}^{* *}$ lies, since if the domain is chosen at random, it could not contain $\bar{x}^{* *}$ or cut it, and in such case $\left\|F^{\prime \prime}(\bar{x})\right\|_{\infty}$ is not bounded. We see in this example that both situations are covered by Theorem 4.5 and in the first one, where the Newton-Kantorovich theorem also covers it, we improve the domains of existence and uniqueness of solution and the a priori error estimates that are obtained from the Newton-Kantorovich theorem.

We begin with the case where $\Omega=B(0, r) \cap \Lambda$ with $r \in\left(r_{1}, r_{2}\right)$. For example, we take $r=\frac{3}{2}$ and choose, as it is usually done $[8], \bar{x}_{0}=(1,1, \ldots, 1)^{T}$ as starting point. So,

$$
\beta=1.6854 \ldots, \quad \eta=0.2349 \ldots, \quad k=1.2255 \ldots, \quad k \beta \eta=0.4853 \ldots \leq \frac{1}{2} .
$$


TABLE 2. Numerical solution of (5.4).

\begin{tabular}{|c|c|c|c|}
\hline$i$ & $x_{i}^{*}$ & $i$ & $x_{i}^{*}$ \\
\hline 1 & $1.019929 \ldots$ & 5 & $1.276373 \ldots$ \\
2 & $1.096786 \ldots$ & 6 & $1.200456 \ldots$ \\
3 & $1.200456 \ldots$ & 7 & $1.096786 \ldots$ \\
4 & $1.276373 \ldots$ & 8 & $1.019929 \ldots$ \\
\hline
\end{tabular}

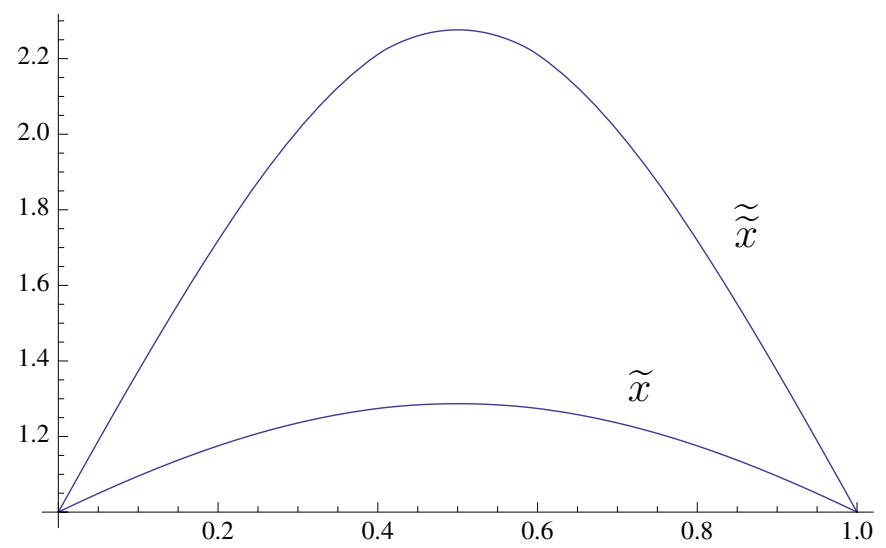

FiguRE 3. Approximated solutions of equation (5.4).

Therefore, from the Newton-Kantorovich theorem, the semilocal convergence of Newton's method to a solution $\bar{x}^{*}$ in $\Omega=B(0, r) \cap \Lambda$ is guaranteed. Taking into account that $p(s)=(0.1394 \ldots)-(0.5933 \ldots) t+(0.6127 \ldots) t^{2}$, $s^{*}=0.4013 \ldots, s^{* *}=0.5669 \ldots$ and $B\left(\bar{x}_{0}, s^{*}\right) \subseteq \Omega$, we obtain that the domains of existence and uniqueness of solutions are respectively

$$
\left\{\bar{\nu} \in \Omega ;\|\bar{\nu}-\overline{1}\|_{\infty} \leq 0.4013 \ldots\right\} \quad \text { and } \quad\left\{\bar{\nu} \in \Omega ;\|\bar{\nu}-\overline{1}\|_{\infty}<0.5669 \ldots\right\} .
$$

On the other hand, if we choose $t_{0}=0$ for Theorem 4.5 , we have $M=0.8340 \ldots, \alpha=0.5479 \ldots$,

$$
\phi(t)=(0.1394 \ldots)-(0.5933 \ldots) t+(0.4170 \ldots) t^{2}+(0.0247 \ldots)^{\frac{5}{2}}+(0.1235 \ldots) t^{3}
$$

and $\phi(\alpha)=-0.0346 \ldots \leq 0$, so that the semilocal convergence of Newton's method to a solution $\bar{x}^{*}$ in $\Omega=$ $B(0, r) \cap \Lambda$ is also guaranteed from Theorem 4.5. Taking into account this and $t^{*}=0.3119 \ldots$ and $t^{* *}=0.7729 \ldots$, we obtain that the the domains of existence and uniqueness of solution are respectively

$$
\left\{\bar{\nu} \in \Omega ;\|\bar{\nu}-\overline{1}\|_{\infty} \leq 0.3119 \ldots\right\} \quad \text { and } \quad\left\{\bar{\nu} \in \Omega ;\|\bar{\nu}-\overline{1}\|_{\infty}<0.7729 \ldots\right\} .
$$

Observe that we improve the domains obtained from the Newton-Kantorovich theorem, since the domain of existence of solution is smaller and the domain of uniqueness of solution is bigger.

Once the convergence of Newton's method is guaranteed, we apply the method to obtain the approximation $\bar{x}^{*}=\left(x_{1}^{*}, x_{2}^{*}, \ldots, x_{8}^{*}\right)^{T}$, given in Table 2, after five iterations. By interpolating the values given in Table 2 and taking into account that equation (5.4) satisfies $x(0)=x(1)=1$, we obtain the solution drawn in Figure 3 and denoted by $\widetilde{x}$. Observe that $\left\|\bar{x}^{*}\right\|_{\infty}=1.2763 \ldots \leq r=\frac{3}{2}$.

Next, we see that the corresponding majorizing sequence given by (3.1) provides better a priori error estimates than those obtained from the majorizing sequence $\left\{s_{n}\right\}$ obtained by the Newton-Kantorovich theorem. The 
TABLE 3. Absolute error and a priori error estimates.

\begin{tabular}{|c|c|c|c|}
\hline$n$ & $\left\|\bar{x}^{*}-\bar{x}_{n}\right\|$ & $\left|t^{*}-t_{n}\right|$ & $\left|s^{*}-s_{n}\right|$ \\
\hline 0 & $0.276373 \ldots$ & $0.311999 \ldots$ & $0.401357 \ldots$ \\
1 & $0.041380 \ldots$ & $0.077005 \ldots$ & $0.166363 \ldots$ \\
2 & $0.001366 \ldots$ & $0.008614 \ldots$ & $0.055542 \ldots$ \\
3 & $1.6 \ldots \times 10^{-6}$ & $0.000139 \ldots$ & $0.011150 \ldots$ \\
\hline
\end{tabular}

a priori error estimates and the absolute error are shown in Table 3. Observe the remarkable improvement obtained from the majorizing sequence constructed in this paper.

On the other hand, we have seen previously that equation (5.4) may have a discrete solution $\bar{x}^{* *}$ such that $\left\|\bar{x}^{* *}\right\| \geq r_{2}=1.6992 \ldots$, but we cannot guarantee the convergence of Newton's method from the Newton-Kantorovich theorem, since we cannot fix a domain that contains $\bar{x}^{* *}$ and where $\left\|F^{\prime \prime}(\bar{x})\right\|_{\infty}$ is bounded. However, from Theorem 4.5, we can do it.

We choose for example the starting vector $\bar{x}_{0}=(3,3, \ldots, 3)^{T}$ and observe that $\left\|\bar{x}_{0}\right\|_{\infty}=3>r_{2}=1.6992 \ldots$ At first, we cannot apply Theorem 4.5 either, since $\phi(\alpha)=0.2910 \ldots>0$ with $\alpha=0.0622 \ldots$ and

$$
\phi(t)=(0.2957 \ldots)-(0.1507 \ldots) t+(1.1922 \ldots) t^{2}+(0.0247 \ldots) t^{\frac{5}{2}}+(0.1235 \ldots) t^{3},
$$

$\beta=6.6347 \ldots, \eta=1.9620 \ldots$ and $M=2.3845 \ldots$ However, it seems clear that improving the initial approximation, the conditions of Theorem 4.5 hold. Indeed, applying Newton's method from $\bar{x}_{0}=(3,3, \ldots, 3)^{T}$, after three iterations, we obtain the vector $\bar{x}_{3}$, given by

$$
\bar{x}_{3}=\left(\begin{array}{l}
1.077814 \ldots \\
1.392262 \ldots \\
1.866018 \ldots \\
2.267211 \ldots \\
2.267211 \ldots \\
1.866018 \ldots \\
1.392262 \ldots \\
1.077814 \ldots
\end{array}\right),
$$

that satisfies, as new starting point, the conditions of Theorem 4.5 , since if we take $\widetilde{\bar{x}}_{0}=\bar{x}_{3}$, we obtain $\beta=2.9176 \ldots, \eta=0.0423 \ldots, M=1.8203 \ldots$,

$$
\phi(t)=(0.0145 \ldots)-(0.3427 \ldots) t+(0.9107 \ldots) t^{2}+(0.0247 \ldots) t^{\frac{5}{2}}+(0.1235 \ldots) t^{3},
$$

$\alpha=0.1791 \ldots$ and $\phi(\alpha)=-0.0166 \ldots \leq 0$. Besides, $t^{*}=0.0487 \ldots, t^{* *}=0.3071 \ldots$ and the domains of existence and uniqueness of solution are respectively

$$
\left\{\bar{\nu} \in \Lambda ;\|\bar{\nu}-\overline{3}\|_{\infty} \leq 0.0487 \ldots\right\} \quad \text { and } \quad\left\{\bar{\nu} \in \Lambda ;\|\bar{\nu}-\overline{3}\|_{\infty}<0.3071 \ldots\right\} .
$$

Observe that the new starting point $\widetilde{\bar{x}}_{0}$ is such that $\left\|\widetilde{\bar{x}}_{0}\right\|_{\infty}=2.2672 \ldots>r_{2}=1.6992 \ldots$ After four more iterations of Newton's method, we obtain the approximated solution $\bar{x}^{* *}=\left(x_{1}^{* *}, x_{2}^{* *}, \ldots, x_{8}^{* *}\right)^{T}$ given in Table 4 , which is a solution that is beyond the scope of the Newton-Kantorovich theorem.

By interpolating the values of Table 4 and taking into account again that the solutions of (5.4) satisfy $x(0)=x(1)=1$, we obtain the solution drawn in Figure 3 and denoted by $\widetilde{\widetilde{x}}$. 
TABLE 4. Numerical solution of (5.4).

\begin{tabular}{|c|c|c|c|}
\hline$i$ & $x_{i}^{* *}$ & $i$ & $x_{i}^{* *}$ \\
\hline 1 & $1.075236 \ldots$ & 5 & $2.222620 \ldots$ \\
2 & $1.379101 \ldots$ & 6 & $1.836355 \ldots$ \\
3 & $1.836355 \ldots$ & 7 & $1.379101 \ldots$ \\
4 & $2.222620 \ldots$ & 8 & $1.075236 \ldots$ \\
\hline
\end{tabular}

\section{Conclusions}

The main aim of this paper is to modify the domain of valid starting points for Newton's method with respect to that given by Kantorovich, so that we can guarantee the semilocal convergence of Newton's method from starting points that the well-known Newton-Kantorovich theorem cannot. For this, we require stronger conditions to the operator involved than Kantorovich's ones. In particular, centered conditions are required to the second derivative of the operator involved.

Moreover, we improve the domains of existence and uniqueness of solution and the a priori error bounds. We also mention a result on the speed of convergence as published elsewhere and see that some results from the literature can be derived as special cases of our results. Finally, we show some applications of our results to solving nonlinear integral equations, which cannot be solved using the Newton-Kantorovich theorem.

Acknowledgements. This work was supported in part by the project MTM2011-28636-C02-01 of the Spanish Ministry of Science and Innovation.

\section{REFERENCES}

[1] S. Amat and S. Busquier, Third-order iterative methods under Kantorovich conditions. J. Math. Anal. Appl. 336 (2007) 243-261.

[2] S. Amat, C. Bermúdez, S. Busquier and D. Mestiri, A family of Halley-Chebyshev iterative schemes for non-Fréechet differentiable operators. J. Comput. Appl. Math. 228 (2009) 486-493.

[3] I.K. Argyros, A Newton-Kantorovich theorem for equations involving $m$-Fréchet differentiable operators and applications in radiative transfer. J. Comput. Appl. Math. 131 (2001) 149-159.

[4] I.K. Argyros, An improved convergence analysis and applications for Newton-like methods in Banach space, Numer. Funct. Anal. Optim. 24 (2003) 653-572.

[5] I.K. Argyros, On the Newton-Kantorovich hypothesis for solving equations. J. Comput. Appl. Math. 169 (2004) $315-332$.

[6] D.D. Bruns and J.E. Bailey, Nonlinear feedback control for operating a nonisothermal CSTR near an unstable steady state. Chem. Eng. Sci. 32 (1977) 257-264.

[7] K. Deimling, Nonlinear functional analysis. Springer-Verlag, Berlin (1985).

[8] J.A. Ezquerro and M.A. Hernández, Generalized differentiability conditions for Newton's method. IMA J. Numer. Anal. 22 (2002) 187-205.

[9] J.A. Ezquerro and M.A. Hernández, On an application of Newton's method to nonlinear operators with $\omega$-conditioned second derivative. BIT 42 (2002) 519-530.

[10] J.A. Ezquerro and M.A. Hernández, Halley's method for operators with unbounded second derivative. Appl. Numer. Math. 57 (2007) 354-360.

[11] J.A. Ezquerro, D. González and M.A. Hernández, Majorizing sequences for Newton's method from initial value problems. J. Comput. Appl. Math. (submitted).

[12] M. Ganesh and M.C. Joshi, Numerical solvability of Hammerstein integral equations of mixed type. IMA J. Numer. Anal. 11 (1991) 21-31.

[13] J.M. Gutiérrez, A new semilocal convergence theorem for Newton's method. J. Comput. Appl. Math. 79 (1997) $131-145$.

[14] L.V. Kantorovich, On Newton's method for functional equations. Dokl Akad. Nauk SSSR 59 (1948) 1237-1240 (in Russian).

[15] L.V. Kantorovich, The majorant principle and Newton's method. Dokl. Akad. Nauk SSSR 76 (1951) 17-20 (in Russian).

[16] L.V. Kantorovich and G.P. Akilov, Functional analysis. Pergamon Press, Oxford (1982).

[17] A.M. Ostrowski, Solution of equations in Euclidean and Banach spaces. London, Academic Press (1943). 
[18] F.A. Potra and V. Pták, Sharp error bounds for Newton process. Numer. Math. 34 (1980) 63-72.

[19] J. Rashidinia and M. Zarebnia, New approach for numerical solution of Hammerstein integral equations. Appl. Math. Comput. 185 (2007) $147-154$.

[20] W.C. Rheinboldt, A unified convergence theory for a class of iterative processes. SIAM J. Numer. Anal. 5 (1968) $42-63$.

[21] T. Yamamoto, Convergence theorem for Newton-like methods in Banach spaces. Numer. Math. 51 (1987) 545-557.

[22] Z. Zhang, A note on weaker convergence conditions for Newton iteration. J. Zhejiang Univ. Sci. Ed. 30 (2003) 133-135, 144 (in Chinese). 